\title{
The association of carcinoma, tuberculosis, asbestosis, and fibrosing alveolitis of the lung: Report of a case and review of the literature
}

\author{
C. IVAN GORDON, D.O., FCAP, FASCP \\ Cherry Hill, New Jersey
}

\section{This paper reports on an} unusual combination of four associated diseases, namely, carcinoma, tuberculosis, asbestosis, and fibrosing alveolitis. Various combinations of the first three have been reported in pairs; however, it is rare and unusual to find all of the first three diseases in one patient simultaneously or sequentially. Even more unusual is the association of the first three with the fourth, fibrosing alveolitis. This present clinical experience raises the question of why a patient with proven tuberculosis should wax and wane despite conventional adequate therapy. Perhaps one answer maybe to consider such patients as having multiple associated disease entities that impact negatively upon tubercular therapy.

The association of tuberculosis with carcinoma has been known for many decades ${ }^{1,2}$ and has been reported numerous times. In addition, the association of asbestosis with carcinoma ${ }^{3-6}$ and asbestosis with tuberculosis ${ }^{7}$ is also well documented. However, a diligent search of the literature dating back to 1960 revealed only two reports of the presence of all three diseases, that is, asbestosis, carcinoma, and tuberculosis in a single patient.

The case presented here describes a patient with this triad as well as fibrosing alveolitis, a combination that was not discovered in the literature search.

\section{Report of case \\ Clinical presentation}

A 61-year-old Caucasian man was admitted on January 31,1986 , with a diagnosis of recurrent, resistant, typical tuberculosis and a chief complaint of shortness of breath. The patient had recently been discharged from an area hospital, where he had been admitted for severe dyspnea, a productive cough of white sputum, and anorexia with severe weight loss. He had been treated at other institutions until this admission.

Further history taking revealed that in October 1977, the patient was evaluated for a left apical lesion associated with pleuritic pain and a small effusion. Bronchoscopy, thoracentesis, and, at a later date, pleural biopsy all were negative for tuberculosis. However, the PPD tuberculin test was positive, with a negative histoplasmin reaction. He was treated for tuberculosis with Isoniazid (INH) and Ethambutol for 1 year, and the lesion stabilized to fibrotic strands. In January 1983, a left apical cavity appeared, and the patient became notably dyspneic. Repeat bronchoscopy and subsequent cultures were positive for tuberculosis organisms, which were resistant to INH and para-aminosalicylic acid (PAS). Nevertheless, he was given INH and Ethambutol; Rifampin subsequently was added, and the cavitary lesion improved. In December 1984, almost 2 years later, the lesion appeared to have worsened. Bronchoscopy was repeated in February or March 1985, and subsequent acid-fast bacillus cultures were negative initially. By April 1985, the cultures were reported to be positive for Mycobacterium tuberculosis but were resistant to the usual therapy. In May 1985, Capreomycin was started intramuscularly and continued for 6 months, until November 1985. He also was treated with Pyrazinamide $(500 \mathrm{mg}$. 3 times a day), as well as Ethambutol ( $800 \mathrm{mg}$. daily) and Rifampin ( $600 \mathrm{mg}$. daily). Initially, the patient became less dyspneic, but he then became anorexic and experienced gradually increasing dyspnea as well as onset of angina. Right pleural effusion now appeared, which sub- 
sequently cleared.

His past medical history revealed coronary artery ischemic disease, and subsequent cardiac catheterization revealed a restrictive type of cardiomyopathy of unknown etiology. The ejection fraction was 27 percent. Furthermore, he was known to have chronic obstructive lung disease, hypertension, and noninsulin-dependent diabetes. He had undergone previous throat surgery of an unspecified nature, and had been diagnosed as having a psychosis (unspecified type). His work history revealed that he was a pipe fitter for 25 years. The social history noted the patient to have been a smoker of 1 packs of cigarettes per day over a 45-year period. He was known to use alcohol.

The physical examination revealed a cachetic-appearing man who was alert and in no apparent distress. His vital signs were as follows: blood pressure, $110 / 80 \mathrm{~mm}$. $\mathrm{Hg}$; heart rate, 80 beats/min.; respiratory rate, $22 / \mathrm{min}$.; and temperature, $97.6 \mathrm{~F}$. The head, eyes, ears, nose, and throat were unremarkable. The posterior pharynx was slightly hyperemic. The neck was supple, with no jugular venous distentions or bruits. The lungs demonstrated symmetric excursions, with fine, dry, crackling rales at the left base. S1 and S2 heart sounds were noted; a grade II/VI systolic ejection murmur at the apex was nonradiating, and no heaves were appreciated. The abdomen was flat, soft, and nontender, and there were no masses or organomegaly. Bowel sounds were satisfactory. The extremities failed to demonstrate clubbing, cyanosis, or edema. A $3+$ dorsalis pedis pulse was palpated bilaterally.

Laboratory findings on admission revealed a leukocyte count of $10,500 / \mathrm{cu}$. mm., with a normal differential distribution. The hemoglobin concentration was $12.3 \mathrm{gm}$./ dl., the hematocrit reading was 38 percent, the $\mathrm{MCH}$, $\mathrm{MCHC}$, and MCV were normal, and the platelet count was $363,000 /$ cu.mm. Urinalysis had normal results. The SMAC battery had normal values, except for a low total protein level of $5.7 \mathrm{gm} . / \mathrm{dl}$. and a high uric acid value of $10.9 \mathrm{mg} . / \mathrm{dl}$. The blood type was $\mathrm{O}$ positive. Prothrombin and partial thromboplastin times were normal. The chest $\mathrm{x}$-ray was interpreted to reveal bilateral, upper-lobe granulomatous disease with probable cavitary component, which was compatible with a diagnosis of pulmonary tuberculosis. The electrocardiogram demonstrated multifocal atrial tachycardia with prominent voltage, possible right ventricular hypertrophy, and nonspecific ST-T wave abnormalities.

The hospital course was initiated by placing the patient in isolation because of active pulmonary tuberculosis. Several sputum specimens were obtained; the smears were positive for acid-fast bacilli, and after 6 weeks the cultures were positive. He was treated with Pyrazinamide, INH, Ethionamide, Cycloserine, and Streptomycin. He was continued on Diabinese and Digoxin, as well as on Persantine, Nitroglycerin paste, Lasix, and Cardizem. The patient demonstrated considerable rhythm disturbances, with 24-hour Holter monitor showing intermittent atrial fibrillation, intermittent sinus rhythm with occasional episodes of seconddegree, Mobitz type II heart block, and frequent premature atrial beats (often nonconducted) as well as pre- mature ventricular beats with one triplet. However, none of these rhythm disturbances correlated with symptoms. The Cardizem therapy was changed to Verapamil, which resulted in better control of the arrhythmias. Although the patient had episodic chest pain, there were no significant electrocardiographic changes. He developed right pleural effusion, which was tapped and classified as a transudate free of acid-fast bacilli. The effusion was secondary to congestive heart failure and was consistent with the history of compromised left ventricular function. Serial chest $x$-rays showed progression of the infiltrate in the right lung zone, which was thought to represent advancing tuberculosis in spite of therapy.

The patient complained of shortness of breath and required 30-percent oxygen by face mask in order to keep his oxygen partial pressure $\left(\mathrm{PO}_{2}\right)$ at a reasonable level. He was given nebulizer treatments with Alupent several times a day and was placed on Theophylline therapy for a short period of time; this, however, aggravated the arrhythmias and was discontinued. As a result of his multiple medications, there was a rise in most liver enzyme values, with elevations of lactic dehydrogenase, serum glutamic-oxaloacetic transaminase, and alkaline phosphatase. The serum glutamic-pyruvic transaminase value remained normal. There was no electrolyte disturbance. Despite antitubercular therapy, the patient continued to produce modest amounts of acid-fast bacilli in his sputum. The cytology of the pleural fluid was negative. The patient's appetite continued to be poor, he continued to lose weight, and, subsequently, he was started on peripheral parenteral nutrition with 10 percent interlipids.

On February 19, 1986, the patient began developing increasing amounts of dyspnea, tachycardia, and respiratory distress. Arterial blood determinations revealed a ph of $7.11, \mathrm{PCO}_{2}$ of $97 \mathrm{~mm}$./Hg., $\mathrm{PO}_{2}$ of $32 \mathrm{~mm}$. Hg., and a bicarbonate level of 31 . The patient died on February 19, 1986. The final clinical diagnoses were: (1) end-stage pulmonary tuberculosis resistant to multiple drugs; (2) coronary artery disease with angina pectoris; (3) congestive heart failure; (4) diabetes mellitus, and (5) progressive respiratory failure leading to death.

\section{Pathologic evaluation}

The external examination of the body revealed a mature, adult, Caucasian male with extreme cachexia. The patient weighed less than 100 pounds and measured $173 \mathrm{~cm}$. in length. The physiologic state of the body appeared older than the stated chronologic age of 61 years. Extreme loss of tissue turgor together with loss of subcutaneous fat had resulted in dependency of tissue about the upper and lower extremities. The rib cage was outlined. The abdomen was flat. There were no palpable masses and no ballotable fluids.

On internal examination, the heart weighed $450 \mathrm{gm}$. Both left and right ventricular walls were thickened and measured $1.7 \mathrm{~cm}$. and $0.8 \mathrm{~cm}$., respectively. The tricuspid valve measured $10 \mathrm{~cm}$. and the mitral valve $13 \mathrm{~cm}$. in circumference. The wall of the left ventricle was concentrically hypertrophied, and the ventricular cavity was reduced in volume.

The left lung was adherent to the anterior lateral and 
posterior chest walls, and it was separated with great difficulty. There were bilateral multiple, enlarged, fused paratracheal and peribronchial lymph nodes, which on sectioning oozed a soft, creamy, cottage-cheese-like material. On average, these measured 4 by $5 \mathrm{~cm}$. and were soft, with alternating white and anthracotic areas. The pleural surfaces were markedly anthracotic, and there was an ill-defined cavity at the apical portion of the left upper lobe. This area had numerous fibrinous and fibrous adhesions to the chest wall and was separated with great difficulty. The right lung was encased by a thickened, gray-white pleura. The lung itself was markedly edematous. The right lung weighed $800 \mathrm{gm}$., and the left lung weighed $450 \mathrm{gm}$. The cut surface of the left lung demonstrated a honeycomb pattern, while the cut surfaces of the right lung demonstrated milky-white areas dominating the upper lobe.

The right kidney weighed $110 \mathrm{gm}$., and the left kidney $125 \mathrm{gm}$. The capsule of each kidney was stripped with great difficulty. The external surfaces demonstrated multiple Y- and U-shaped scars. There was severe granularity as well as congestion. There was a reduction in cortical thickness.

Sections of the right lung demonstrated a large, infiltrative neoplasm that consisted of sheets of epithelial cells. Some of these sheets formed a mosaic pattern with sharp cell borders. Epithelial bridges were found together with keratinization. Focal as well as diffuse necrotic areas were observed. There was increased mitotic activity. Invasion of venous channels were noted (Fig. 1). In addition, collections of malignant cells within alveoli were observed. There were broad areas of atelectasis. The left lung demonstrated multiple broad, large, caseous granulomas, with centers that were relatively soft and very cellular, and a cortical portion that was eosinophilic. These areas were amorphic and revealed coagulation necrosis (Fig.2). Multinucleated giant cells along the cortical borders were observed. AFB stains on numerous sections were negative. The enlarged paratracheal lymph nodes were tumor bearing.

In addition, emphysematous alterations characterized by interruption of alveolar septae and coalescence of alveolar spaces were observed.

Also observed on the AFB-stained sections were asbestos bodies (Fig. 3), which were characterized by elongated fibers consisting of uniform segments, with clear areas between each segmented portion.

Sections of the kidneys from the scarred V- and Ushaped areas demonstrated numerous obsolescent glomeruli, periglomerular fibrosis, and dilatation of Bowman's capsule, which was occupied by mildly eosinophilic, ground-glass, colloid-like material. In addition, there was severe glomerular congestion. A few proximal tubules demonstrated crystals that were basophilic. Many proximal tubules were dilated and had undergone thyroidization. The vascular system was diffusely congested. There was thickening of the intima and media of the arterioles, with overall reduction of luminal diameters.

\section{Discussion}

The autopsy of this 61-year-old patient with a long-stand-

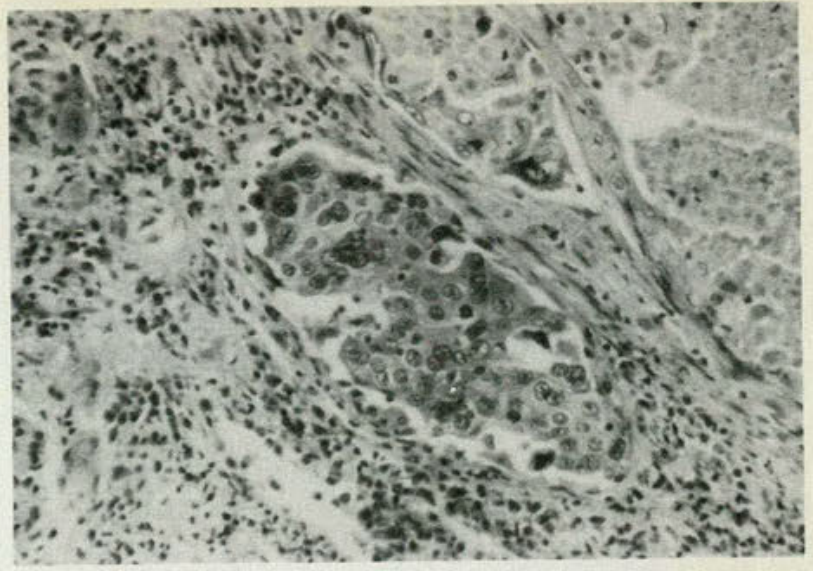

Fig. 1. Squamous cell carcinoma, with stroma of fibrosing alveolitis (hematoxylin and eosin stain, $\times 4$ magnification).

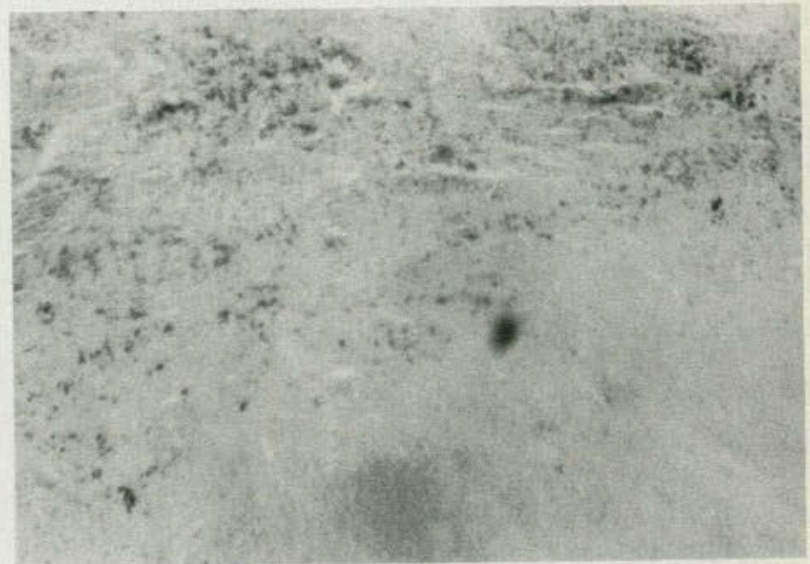

Fig. 2. Caseating granuloma of mycobacterial tuberculosis (hematoxylin and eosin stain, $\times 4$ magnification).

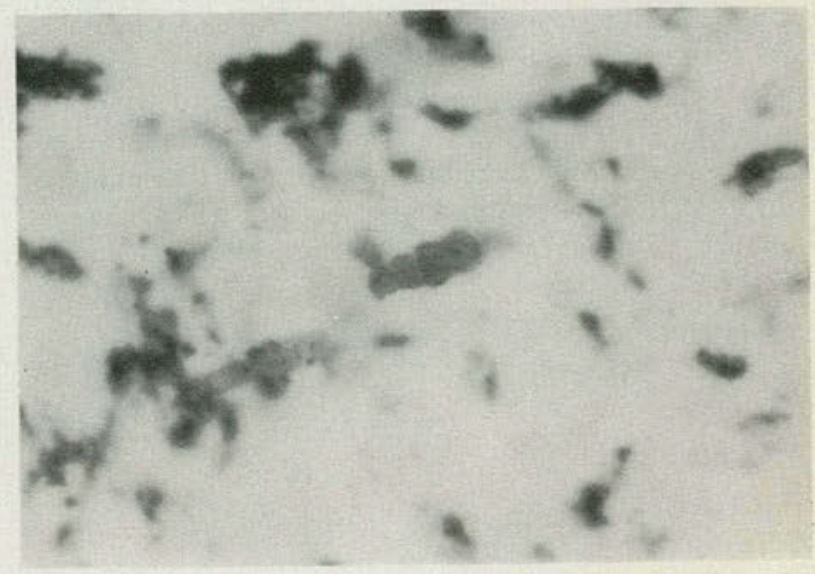

Fig. 3. Asbestos body (hematoxylin and eosin stain, $\times 40$ magnification).

ing history of resistant tubeculosis revealed numerous caseating granulomas in the left upper lobe of the lung. Many AFB sections, however, failed to reveal acid-fast organisms. Subsequent laboratory smears and cultures were positive. In addition, the right lung demonstrated well-differentiated squamous cell carcinoma, which had metastasized to peribronchial and paratracheal lymph nodes. Furthermore, asbestos bodies were found in the 
left lung, together with granulomata as well as fibrosing alveolitis.

The search of the literature for an association of all three clinical entities revealed only one article ${ }^{8}$ which reported 4 cases of asbestosis combined with bronchiogenic carcinoma and pulmonary tuberculosis. One of the 4 cases demonstrated asbestosis along with bacteriologic and cytologic evidence of mycobacterial tuberculosis and undifferentiated squamous cell carcinoma. This was principally a hilar lesion, and it was suspected that the hilar process was tubercular rather than malignant, because there was a response to antitubercular therapy. The second case involved a man with moderately severe asbestosis who subsequently developed pulmonary tuberculosis. He later produced sputum that was suspicious for malignancy, and some 2 years later he was radiologically diagnosed as having carcinoma in the left hilar area. A tissue diagnosis was never obtained. Autopsy was denied, and the diagnosis was inferred from the above evidence. The third case of asbestosis and tuberculosis involved carcinoma of the stomach. The fourth case reported asbestosis, with the patient having 10 years of exposure to asbestos dust. Rapidly progressive bilateral tuberculosis of the upper lobes with cavitation in the left lobe subsequently developed. Bronchiogenic carcinoma was established both histologically and bronchoscopically. These findings were confirmed by autopsy. Thus, of the 4 cases reported, 1 was carcinoma of the stomach, 1 was inferred bronchiogenic carcinoma, and, in reality, only 2 were proved cases of existing pulmonary tuberculosis, asbestosis, and carcinoma of the lung. Therefore, to the author's knowledge, the present case is the third reported incidence of the triad. The short fibers of asbestos are considered to be more dangerous as a carcinogen than are the long fibers. The asbestos fibers tend to be deposited in a peripheral location; there is early involvement of the pleura, more commonly in the lower than the upper lobes. The origin of asbestos-induced tumors follows the asbestos distribution anatomically, and the predominant cell type is adenocarcinoma. However, when asbestos exposure is combined with a history of smoking, the incidence of carcinoma of the lung increases dramatically, and it tends to be of a squamous or oat cell variety, in contrast to the peripheral adenocarcinoma. Also, the peripheral distribution of the asbestos fibers in the pleura has been established as one of the etiologic agents of malignant mesothelioma. ${ }^{8}$

\section{Comment}

The patient in the present case showed great resistance to antitubercular therapy, which signaled that this was more than a routine occurrence of pulmonary tuberculosis. It would appear that a common thread in the reported cases is the rapid progression and death of patients with the associated diseases. Therefore, it should be emphasized that in light of modern tubercular chemotherapy, failure of the patient to respond adequately should raise the suspicion of associated disease, in particular asbestosis and carcinoma.

1. Gloyne, S.R.: Two cases of squamous cancer of the lung occurring in asbestosis. Tubercule 17:5-10, Oct 35

2. Homburger, F.: The co-incidence of primary cancer of the lungs in pulmonary asbestosis. Am J Pathol 19:797-805, 1943

3. Dahl, R.: Mortality from lung cancer in asbestosis workers. Br J Ind Med 12:81-6, 1955

4. Wagner, J.C.: Diffuse pleural mesothelioma in asbestosis exposure in the Northwestern Cape Province. Br J Ind Med 17:260-71, 1960

5. Telischi, M., and Rubestone, A.I.: Pulmonary asbestosis associated with cancer of the lung. Arch Pathol 72:234-43, 1970

6. Merewether, A.R.A.: The occurrence of pulmonary fibrosis and other pulmonary affections in asbestos workers. J Ind Hygiene 12:253-57, 1930 7. Wood, W.B., and Gloyne, S.R.: Pulmonary asbestosis complicated by pulmonary tuberculosis. Lancet 2:954-6, Oct 31

8. Wyers, H., Asbestosis. Postgrad Med J 25:631-8, Dec 49

9. von Arnin, H.H. Asbestose, Bronchialkarzinom und Lungentuberkulose. Prax Pneumol 27:353-8, Jun 73

Dr. Gordon is an associate pathologist, Deborah Heart and Lung Center, Browns Mills, New Jersey.

Dr. Gordon, 1233 Crane Road, Cherry Hill, New Jersey 08003. 